\title{
DIRECT DECOMPOSITIONS OF GROUPS WITH FINITELY GENERATED COMMUTATOR QUOTIENT GROUP
}

\author{
RONALD HIRSHON \\ (Received 30 June 1978; revised 12 April 1979) \\ Communicated by $\mathbf{H}$. Lausch
}

\begin{abstract}
Let $G / G^{\prime}$ be finitely generated and let $G=B_{1} \times A_{1}=B_{2} \times A_{2}=\ldots=B_{i} \times A_{i}=\ldots$ with each $B_{i}$ isomorphic to a fixed group $B$ which obeys the maximal condition for normal subgroups. Then the $A_{i}$ represent only finitely many isomorphism classes. We give an example with $B$ infinite cyclic, $G / G^{\prime}$ free abelian of infinite (countable) rank and such that $G$ is decomposed as above with no two $A_{i}$ isomorphic.
\end{abstract}

1980 Mathematics subject classification (Amer. Math. Soc.): 20 F 06, 20 E 22, 20 E 15.

\section{Introduction}

If $A$ and $B$ satisfy $A \times J \approx B \times J, J$ infinite cyclic, then $A$ and $B$ have the same finite homomorphic images (Hirshon (1977)). It is easy to choose $A \not B, A$ finitely generated and $A \times J \approx B \times J$ (see Hirshon (1969), Baumslag (1977)). These observations lead to a straightforward way of constructing two finitely generated nonisomorphic groups with the same finite homomorphic images. In certain cases $A$ and $B$ can be made to have other prescribed conditions (for example, $A$ and $B$ can be finitely generated torsion-free nilpotent (Hirshon (1977)).

P. Pickel (1974) has constructed an infinitude of isomorphism classes of finitely generated metabelian groups all of which have the same finite homomorphic images. It seems natural to wonder if the ideas above can lead to results like Pickel's. In particular, we ask: When can a group $G$ have infinitely many decompositions

$$
G=\left\langle w_{1}\right\rangle \times A_{1}=\left\langle w_{2}\right\rangle \times A_{2}=\ldots=\left\langle w_{i}\right\rangle \times A_{i}=\ldots
$$


with each $\left\langle w_{i}\right\rangle \approx J$ and no two $A_{i}$ isomorphic? The main purpose of this paper is to show that if $G / G^{\prime}$ is finitely generated then in a sequence of decompositions (1) of $G$, the $A_{i}$ must represent finitely many isomorphism classes. In particular, this holds if $G$ is finitely generated or if $G$ obeys the maximal condition for normal groups.

The above question (1) has, in many cases, a strong connection to the more general question: When can a group $G$ have infinitely many decompositions

$$
G=B_{1} \times A_{1}=B_{2} \times A_{2}=\ldots=B_{i} \times A_{i}=\ldots
$$

with each $B_{i}$ isomorphic to a fixed group $B$ and no two $A_{i}$ isomorphic? To see the connection, we define a group $B$ to be $J$ replaceable if the isomorphism $C \times B \approx D \times B$ always implies $C \times J \approx D \times J$. For example, a group which obeys the maximal condition for normal subgroups is $J$ replaceable (Hirshon (1977)). Hence if $B$ satisfies the maximal condition for normal subgroups and $G / G^{\prime}$ is finitely generated in (1)*, the $A_{i}$ represent finitely many isomorphism classes. Some more general $J$ replaceable groups are given in Hirshon (1978), (1979).

Finally, we give an example of a sequence (1) in which $G / G^{\prime}$ is free abelian of infinite (countable) rank and the $A_{i}$ represent infinitely many isomorphism classes.

\section{The main result}

THEOREM. Let $G=\left\langle w_{i}\right\rangle \times A_{i}, i \geqslant 1,\left\langle w_{i}\right\rangle \approx J$. Let $G / G^{\prime}$ be finitely generated. Then the $A_{i}$ represent finitely many isomorphism classes.

Proof. We proceed indirectly and suppose that the $A_{i}$ represent an infinite number of distinct isomorphism classes. Without loss of generality, we may suppose that if $i<j$, then $A_{i} \not A_{j}$. Let $A_{i_{j}} 1 \leqslant j<\infty$ be an infinite sequence of distinct $A$ 's and let $L$ be their intersection. One can verify that $G / L$ is a torsion-free abelian group. Hence $G / L$ is a finitely generated free abelian group of rank $r$ where $r$ is at most the rank of the torsion-free part of $G / G^{\prime}$. We choose the $A_{i_{j}}$ so that $r$ is as small as possible. Let $B_{j}=A_{i_{j}}$ and $u_{j}=w_{i_{j}}$. Note that if $K$ represents the intersection of any infinite number of distinct $B$ 's, then $K=L$. For $L \subset K \subset G$ and $G / K$ is a free abelian homomorphic image of $G / L$. If $L \neq K$, the rank of $G / K$ would be less than $r$. If $Z\left(B_{1}\right) \subset L$ then all the $B_{i}$ are isomorphic to $B_{1}$. For example, if $u_{2}=u_{1}^{s} b_{1}, b_{1} \in B_{1}$ then $b_{1}$ is central and $b_{1} \in B_{2}$ so

$$
\left\langle u_{2}\right\rangle \times B_{2}=\left\langle u_{2} b_{1}^{-1} \times B_{2}=\left\langle u_{1}^{s}\right\rangle \times B_{2}=\left\langle u_{1}\right\rangle \times B_{1},\right.
$$

so that $s=1$ or $s=-1$ and $B_{2} \approx B_{1}$. Hence we may suppose that we can find $b_{1} \notin L, b_{1} \in Z\left(B_{1}\right)$. For infinitely many $i$ then $b_{1} \notin B_{i}$. Hence we may assume that 
$b_{1} \notin B_{i}$ for all $i$ (or else we merely change notation). Hence $\left[\left(\left\langle u_{1}\right\rangle \times\left\langle b_{1}\right\rangle\right) Z\left(B_{i}\right)\right] / Z\left(B_{i}\right)$ is isomorphic to $J$. Hence $\left\langle u_{1}\right\rangle \times\left\langle b_{1}\right\rangle=\left\langle g_{i}\right\rangle \times\left\langle b_{i}\right\rangle \approx J \times J$ where

$$
\left\langle b_{i}\right\rangle=Z\left(B_{i}\right) \cap\left(\left\langle u_{1}\right\rangle \times\left\langle b_{1}\right\rangle\right) \text {. Also if } b_{i}=u_{1}^{m_{i}} b_{1}^{n_{i}} \text { then } m_{i} \neq 0
$$

or else $n_{i}$ is 1 or -1 and $b_{1} \in B_{i}$. Clearly $\left(m_{i}, n_{i}\right)=1$.

Note that $r=1$ implies that all the $B_{i}$ are identical. Hence $r \geqslant 2$ and $B_{i} / L$ is of rank $r-1=q$. Let $B_{1}=\left\langle h_{1}\right\rangle\left\langle h_{2}\right\rangle \ldots\left\langle h_{q}\right\rangle L$. Let $b_{1}=h_{1}^{c_{1}} h_{2}^{c_{2}} \ldots h_{q}^{c_{q}}, \bmod L$. Hence

$$
b_{i}=u_{1}^{m_{i}} h_{1}^{n_{i} c_{1}} h_{2}^{n_{i} c_{2}} \ldots h_{q}^{n_{i} c_{q}} l_{i}, \quad l_{i} \in L .
$$

Let $d_{i}$ be the greatest common divisor of the integer $m_{i}, n_{i} c_{1}, n_{i} c_{2}, \ldots, n_{i} c_{q}$. If $d_{i}=1$, then a set of free generators for $G / L$ may be chosen which contains $b_{i} L$. This would imply that $\left\langle b_{i}\right\rangle$ is a direct factor of $G$ and hence a direct factor of $B_{i}$. But then by Lemma 1 of Hirshon (1977) $B_{i} \approx B_{1}$. Hence $d_{i} \neq 1, i>1$. Since $\left(m_{i}, n_{i}\right)=1$, $d_{i}$ is a divisor of each of the $c_{j}$. It follows that we may choose a largest possible divisor $d$ of the $c_{j}, d>1$, such that we may write for infinitely many $i$, that $b_{i}$ has a $d$ th root, $\bmod L$. That is for some $v_{i} \in G$

$$
b_{i}=v_{i}^{d}, \bmod L
$$

Again for ease of notation we may assume that (3) holds for all $i$ (or simply pass to a subsequence and change notation). Let $B_{i}=\left\langle c_{i 1}\right\rangle\left\langle c_{i 2}\right\rangle \ldots\left\langle c_{i q}\right\rangle L$. Consider the group $G_{i}$ generated by $u_{i}^{d}, c_{i l}^{d}, \ldots, c_{i q}^{d}$ and $L . G_{i}$ is of index $d^{r}$ in $G$. Since $G / L$ has only finitely many subgroups of a given index, infinitely many of the $G_{i}$ are identical. The argument that follows will depend on the fact that infinitely many of the $G_{i}$ are identical but not on the nature of the indices $i_{k}$ with $G_{i_{1}}=G_{i_{2}}=\ldots$. Hence to avoid an awkward notation let us suppose that we have

$$
G_{2}=G_{4}=G_{6}=G_{8}=\ldots=G_{2 n}=\ldots
$$

Since $b_{2 j} \in G_{2 j}=G_{2}$, we may write

$$
b_{2 j}=u_{2}^{d e_{j}} c_{21}^{d e_{11}} c_{22}^{d e_{22}} \ldots c_{2 q}^{d e_{i q}}, \bmod L .
$$

Let $f_{j}$ be the greatest common divisor of the integers $e_{j}, e_{j 1}, e_{j 2}, \ldots, e_{j q}$. By comparing (2) and (4) with $i=2 j$ we see $d f_{j}$ is a divisor of the integers $c_{1}, c_{2}, \ldots, c_{q}$. If $f_{j} \neq 1$ for infinitely many $j$, we can find a prime $p$ diving infinitely many $f_{j}$ and (4) may then be written as

$$
b_{2 j}=\left(\tilde{v}_{j}\right)^{d p}, \bmod L, \text { for some } \tilde{v}_{j} \in G,
$$

for infinitely many $j$. Since $d p$ is a divisor of $c_{1}, c_{2}, \ldots, c_{q}$ this contradicts the maximality of $d$. Without loss of generality we assume then that $f_{j}=1$ for all $j$. 
Now let $\bar{B}_{i}$ be the subgroup of $B$ generated by $L$ and $c_{i 1}^{d}, c_{i 2}^{d}, \ldots, c_{i q}^{d}$ so that $G_{i}=\left\langle u_{i}^{d}\right\rangle \times \bar{B}_{i}$. Note that $f_{j}=1$ implies that $\left\langle b_{2 j}\right\rangle$ is a direct factor of $G_{2}$ and hence of $\bar{B}_{2 j}$. Hence from Lemma 1 of Hirshon (1977) we may deduce that $\bar{B}_{2} \approx \bar{B}_{2 j}$ for all $j$. In fact if $Z=Z\left(G_{2}\right)$ one can verify that we can find an isomorphism $\theta_{2 j}$ of $\bar{B}_{2}$ onto $\bar{B}_{2 j}$ with $b^{-1} \theta_{2 j} b \in Z, b \in \bar{B}_{2}$ and such that $\theta_{2 j}$ fixes $L$ pointwise. Hence for each $j$ we may express the generators $c_{2 i}^{d}, 1 \leqslant i \leqslant q$ in terms of the generators $c_{2 i, i}^{d}, 1 \leqslant i \leqslant q(\bmod Z L)$. Note that modulo the subgroup $Z L$ the matrix of exponents connecting these generators is unimodular. After regrouping terms, we end up with for each $j$

$$
c_{2 i}^{d}=u_{j i}^{d} z_{j i} l_{j i}
$$

where $u_{j i}, u_{j 2}, \ldots, u_{j q}$ are free generators of $B_{2 j}, \bmod L$, and $z_{j i} \in Z$ and $l_{j i} \in L$. Now consider $q$-tuplets $\bar{S}=\left(s_{1}, s_{2}, \ldots, s_{q}\right)$ where $s_{i} \in Z$. If $\bar{R}=\left(r_{1}, r_{2}, \ldots, r_{q}\right)$ define $\bar{R} \approx \bar{S}$ if $r_{i}=s_{i}, \bmod Z^{d} L$, for all $i$. Since $G / L$ is finitely generated, the above equivalence relation determines finitely many equivalence classes. Let $\bar{R}_{i}=\left(z_{i 1} z_{i 2} \ldots z_{i q}\right), i \geqslant 1$. Choose fixed integer $p, m, p<m$ and with $\bar{R}_{p} \approx \bar{R}_{m}$. Hence for $1 \leqslant f \leqslant q$, we have $z_{p f}=z_{m f} z_{f}^{d} l_{f}, z_{f} \in Z, l_{f} \in L$. Hence $\left(u_{p f}^{-1} u_{m f} z_{f}^{-1}\right)^{d}=l_{f} l_{p f} l_{m f}^{-1}$. Since $G / L$ is torsion free we have $u_{p f}^{-1} u_{m f} z_{f}^{-1}=\bar{l}_{f} \in L$. Now it is easy to check that the map

$$
u_{p f} \longrightarrow u_{m f}\left(\bar{l}_{f}\right)^{-1}, \quad 1 \leqslant f \leqslant q, \quad l \longrightarrow l, \quad l \in L,
$$

induces an isomorphism of $B_{2 p}$ onto $B_{2 m}$ contrary to the assumption that no two $B$ 's are isomorphic.

We have actually shown somewhat more than the assertion of the theorem. Note that if we have a decomposition (1) with the $A_{i}$ representing infinitely many isomorphism classes then our arguments show that $G / L$ is not finitely generated. But if $Q_{0}=G, Q_{n}=A_{1} \cap A_{2} \cap \ldots \cap A_{n}, n \geqslant 1$, then either $Q_{i}=Q_{i+1}$ or $Q_{i} / Q_{i+1} \approx J$. Also $G / Q_{n}$ is a finitely generated free abelian group of rank at most $n$. Moreover, for infinitely many $i, Q_{i} / Q_{i+1} \approx J$ or else ultimately $Q_{i}=Q_{i+1}$. This would imply that $L=Q_{m}$ for some $m$ contradicting the fact that $G / L$ is not finitely generated. We may state then the

COROLlaRY. If in (1) the $A_{i}$ represent infinitely many isomorphism classes, $G$ has a descending sequence of normal groups $L_{1}, L_{2}, L_{3}, \ldots, L_{n}, \ldots$ with $G / L_{n}$ free abelian of rank $n$.

Proof. Let $L_{1}=Q_{1}=A_{1}$. Suppose $L_{n}$ has been defined with $L_{n}=Q_{i_{n}}$ for some $i_{n}$. Let $i_{n+1}$ be the smallest integer $i_{n+1}>i_{n}$ such that $Q_{i_{n+1}}$ is a proper subgroup of $Q_{n}$. Take $L_{n+1}=Q_{i_{n+1}}$. 


\section{An example}

The idea of the following example may be briefly outlined as follows. We choose an infinite sequence of groups $A_{i}, i=1,2, \ldots$, with no two $A_{j}$ isomorphic and such that $A_{i} \times J \approx B_{i} \times J, A_{i} \not B_{i}$. Let $G$ be the (restricted) Cartesian product $G=A_{1} \times A_{2} \times A_{3} \times \ldots$ Then

$$
\left.\left.J \times G=\left(J \times A_{i}\right) \times \underset{k \neq i}{\left(\times A_{k}\right.}\right) \approx\left(J \times B_{i}\right) \times \underset{k \neq i}{\left(\times A_{k}\right.}\right) \approx J \times G_{i},
$$

where

$$
\left.G_{i}=B_{i} \times \underset{k \neq i}{\left(\times A_{k}\right.}\right)
$$

We will show that we can choose the $A_{i}$ so that if $s \neq t$ then $G_{2} \not G_{t}$.

To construct the $A_{i}$, let $p_{1}, p_{2}, p_{3}, \ldots$ be a sequence of distinct primes with $p_{i} \equiv 3$, $\bmod 8$. Let

$$
A_{i}=\left\langle c_{i}, d_{i} ; c^{-1} d_{i} c_{i}=d_{i}^{2}, d_{i}^{p_{i}}=1\right\rangle
$$

and let

$$
B_{i}=\left\langle e_{i}, f_{i} ; e_{i}^{-1} f_{i} e_{i}=f_{i}^{4}, f_{i}^{p_{i}}=1\right\rangle
$$

Then

$$
A_{i} \times J \approx B_{i} \times J \text { and } A_{i} \not B_{i}
$$

(see Baumslag (1974)).

Using the $A_{i}, B_{i}$ as above, we assert that no two of the $G_{i}$ are isomorphic. For say, for example, that $G_{1} \approx G_{2}$ and $\theta$ is an isomorphism of $G_{2}$ onto $G_{1}$. Clearly we must have $d_{1} \theta=f_{1}^{u},(u, p)=1, p=p_{1}$. Let us write $c_{1} \theta=e_{1}^{h} f_{1}^{j} a$ where $a$ is in $A_{2} \times A_{3} \times \ldots$ But then from $c_{1}^{-1} d_{1} c_{1}=d_{1}^{2}$ we obtain $e_{1}^{-h} f_{1}^{u} e_{1}^{h}=f_{1}^{2 u}$. Hence $h \neq 0$ and by a change in generators if necessary we may assume $h>0$. But the last equality implies $f_{1}^{4^{h} u}=f_{1}^{2 u}$. Hence $4^{h} \equiv 2, \bmod p$. Hence $2^{2 h-1} \equiv 1, \bmod p$. But then if $r$ is the order of $2, \bmod p$, this says that $r$ is a divisor of $2 h-1$ so that $r$ is odd. But since $2^{p-1} \equiv 1, \bmod p, r$ is also a divisor of $p-1$ and hence of $(p-1) / 2$. Hence $2^{(p-1) / 2} \equiv 1, \bmod p$. However, $2^{(p-1) / 2} \equiv-1, \bmod p$ (Hardy and Wright (1960), Theorems 83 and 95 ) a contradiction.

\section{References}

G. Baumslag (1974), 'Residually finite groups with the same finite images', Compositio Math. 29, 249-252.

G. H. Hardy and E. M. Wright (1960), An Introduction to the Theory of Numbers (Clarendon Press, Oxford).

R. HiRshon (1969), ‘On cancellation in groups', Amer. Math. Monthly 76, 1037-1039. 
R. Hirshon (1977), 'Some cancellation theorems with applications to nilpotent groups', $J$. Austral. Math. Soc. (Ser. A) 23, 147-165.

R. Hirshon (1978), 'Cancellation and Hopficity in direct products', J. Algebra 50, 26-33.

R. Hirshon (1979), 'The equivalence of $\times{ }^{t} C \approx \times{ }^{t} D$ and $J \times C \approx J \times D$, Trans. Amer. Math. Soc. 249, 331-340.

P. Pickell (1974), 'Metabelian groups with the same finite quotients', Bull. Austral. Math. Soc. 11, 115-121.

Mathematics Department

Polytechnic Institute of New York

333 Jay Street

Brooklyn, N.Y. 11201

U.S.A. 